\title{
Pleural lavage cytology in non-small cell lung cancer: Lessons from 1000 consecutive resections
}

\author{
Morihito Okada, MD, PhD \\ Toshihiko Sakamoto, MD, PhD \\ Wataru Nishio, MD, PhD \\ Kazuya Uchino, MD \\ Kenji Tsuboshima, MD \\ Noriaki Tsubota, MD, PhD
}

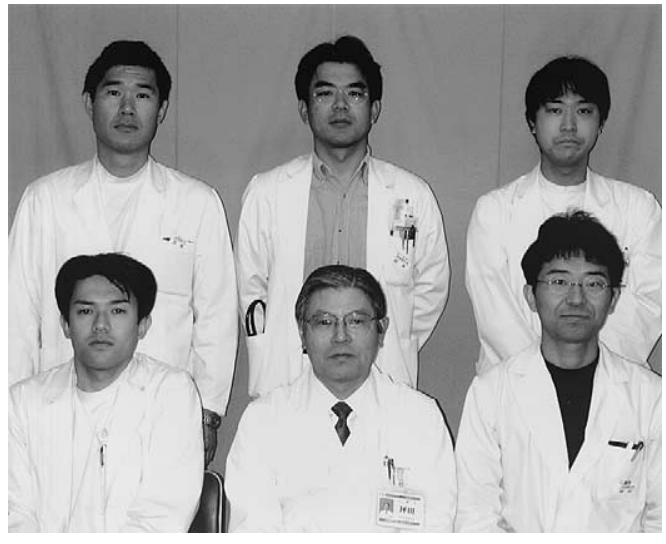

Uchino, Tsubota, Okada (row 1); Nishio, Sakamoto, Tsuboshima (row 2)
Objective: Intraoperative pleural lavage cytology for lung cancer has not been widely accepted. The prognostic significance of this procedure has yet to be intensively analyzed because the reports published thus far have involved small patient populations. We therefore performed a large prospective trial of pleural lavage cytology to elucidate its importance.

Methods: Cytologic status of pleural lavage fluid before any manipulation of the lung was examined in 1000 consecutive patients with non-small cell lung cancer but no pleural effusion who underwent tumor resection.

Results: Forty-five (4.5\%) of 1000 patients had positive cytologic findings. Positive cytologic findings were observed more frequently in patients with adenocarcinoma, advanced stage, higher involvement of lymph nodes, pleural involvement of the tumor, lymphatic permeation, vascular invasion, high level of serum carcinoembryonic antigen, and male sex. The survival rate for 5 years was $28 \%$ in patients with positive findings and $67 \%$ in patients with negative findings $(P<.0001)$. Among 587 patients with stage I disease, $13(2.2 \%)$ had positive findings, and their 5 -year survival was $43 \%$, which was significantly poor compared with that of patients with negative findings $(81 \%, P=.0009)$. Multivariable analysis demonstrated that pleural lavage cytology was an independent prognostic determinant $(P$ $=.0290)$. Regarding the recurrence pattern in patients with positive findings, distant metastases (19/45 [42.2\%]) were observed more frequently rather than local recurrences $(19 / 45[22.2 \%])$.

Conclusions: Cytologic status of pleural lavage fluid immediately after thoracotomy, an independent significant prognostic factor, constitutes valuable information to detect patients at a high risk of recurrence. Therefore cytology should be performed at the time of curative resection for non-small cell lung cancer.

Hyogo Medical Center for Adults, Akashi City, Hyogo, Japan.

Received for publication Jan 12, 2003; revisions requested March 24, 2003; revisions received April 1, 2003; accepted for publication April 8, 2003

Address for reprints: Morihito Okada, MD, $\mathrm{PhD}$, Department of Thoracic Surgery, Hyogo Medical Center for Adults, Kitaohjicho 13-70, Akashi City 673-8558, Hyogo, Japan (E-mail: morihito1217jp@aol.com).

J Thorac Cardiovasc Surg 2003;126:1911-5

Copyright (C) 2003 by The American Association for Thoracic Surgery

0022-5223/2003\$30.00+0

doi:10.1016/S0022-5223(03)00715-3

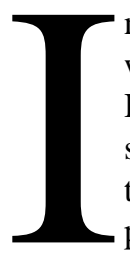

$\mathrm{n}$ general, the presence of malignant cells in pleural effusion of patients with lung cancer is indicative of advanced disease and poor prognosis. However, despite the lack of malignant effusion or dissemination macroscopically, there are instances in which malignant cells might be present in the pleural cavity. ${ }^{1-6}$ In previous reports the rate of positive results of pleural lavage cytology before lung manipulation for non-small cell lung cancer was $4 \%$ to $14 \% .^{1-3,5,7,8}$ The patients with positive lavage cytologic findings, which might indicate an initial stage of carcinomatous pleuritis, have a poor survival rate because a small amount of malignant pleural effusion or a few minute pleural dissemination nodules are probably overlooked at thoracotomy. Cytologic findings of positive lavage fluid without effusion might be ascribed to exfoliation from 
tumors at the pleural surface, thereby representing localized disease, or it might correspond to disseminated disease, implying much more aggressive tumor biology. Currently, cytologic examination of pleural lavage fluid is not routinely performed before resection for lung cancer. The role of this procedure has yet to be intensively investigated because the reports published thus far dealt with small patient populations. The present study, which is a large prospective trial built on our previously reported experience with pleural lavage fluid in patients undergoing resection for lung cancer $^{1}$ and followed for long-term outcome, was undertaken to analyze the survival and the pattern of recurrence and to evaluate the independent prognostic significance of pleural lavage fluid cytology.

\section{Material and Methods}

From January 1987 to December 2001, cytologic status of pleural lavage fluid was examined before any manipulation or resection of the lung in 1000 consecutive patients who had no pleural effusion or diffuse adhesions and who underwent resections for primary non-small cell lung cancer. Informed consent was sought from each patient in regard to participation in this research.

Only when there was no macroscopic pleural effusion was the patient included in this study. Patients with macroscopic pleural effusion that could be collected with a syringe were excluded from this study. Patients who had been subjected to preoperative chemotherapy or radiotherapy were excluded. Preoperative evaluation included a detailed history and physical examination, biochemical profile, chest radiographic examination, bronchoscopy, and computed tomography of the chest, brain, and upper portion of the abdomen. Because there is some fear that preoperative transthoracic needle aspiration biopsy might cause the mechanical exfoliation of cancer cells into the pleural cavity, ${ }^{9}$ we have avoided transthoracic biopsy from the beginning of this study. Seventy-four patients with malignant effusion who underwent resection for primary tumor during the same period but who were excluded from this study served as control subjects for comparison of survival. They had the same clinical background as patients with positive pleural lavage cytologic findings. Immediately after thoracotomy, the pleural cavity was carefully washed with $100 \mathrm{~mL}$ of physiologic saline solution before any further manipulation of the pulmonary parenchyma. The surgeon avoided touching the pleural surface to obtain only desquamated cells. The fluid was then put into a glass bottle with heparin and was centrifuged at $1500 \mathrm{rpm}$ for 5 minutes. The obtained sedimented material was stained with the Giemsa and Papanicolaou methods. The results of the cytologic examination were divided into 2 categories: negative and positive. Papanicolaou classes I to III were regarded as negative, and classes IV to $\mathrm{V}$ were regarded as positive. Pathologic staging was determined according to the international staging system. ${ }^{10}$ Intraoperative staging was done by means of dissecting intrapulmonary, hilar, and mediastinal lymph nodes, and careful postoperative examination was carried out by pathologists. The histologic type of the tumor was determined by applying the World Health Organization classification. The outcomes of histopathologic examination regarding the status of lymph node metastasis, pleural involvement of the tumor, lymphatic permeation by the tumor, and vascular involvement of the tumor were abbreviated as $\mathrm{N}, \mathrm{P}, \mathrm{Ly}$, and $\mathrm{V}$ factors, respectively. These factors were defined as follows: N0, no lymph node metastasis; N1, metastasis in hilar nodes; N2, metastasis in ipsilateral mediastinal nodes; $\mathrm{P}(+)$, tumor has invaded the visceral pleura; $\mathrm{P}(-)$, tumor has not invaded the visceral pleura; $\mathrm{Ly}(+)$, positive lymphatic permeation; $\mathrm{Ly}(-)$, negative lymphatic permeation; $\mathrm{V}(+)$, positive vascular involvement; and $\mathrm{V}(-)$, negative vascular involvement. The normal limit for serum carcinoembryonic antigen (CEA), as assessed by means of immunoenzymometric assay, was $5 \mathrm{ng} / \mathrm{mL}$. From the representative sections stained with hematoxylin and eosin or elastica van Gieson, the presence of tumor cells, including emboli, in endothelial-lined channels of lymphatic and vascular vessels was determined by means of identification of intratumoral lymphatic permeation and vascular involvement, respectively. The Mann-Whitney $U$ test was carried out to investigate the associations between cytologic results of pleural lavage fluid and clinicopathologic factors. Survival was calculated by using the Kaplan-Meier method, and differences in survival were determined by means of log-rank analysis. A multivariable analysis of several prognostic factors was carried out by using the Cox proportional hazards regression model. Zero time was the date of pulmonary resection, and the terminal event was death attributable to cancer, noncancer, or unknown causes.

\section{Results}

Among 1000 patients with non-small cell lung cancer in whom pleural lavage cytologic status was examined, 45 (4.5\%) had positive findings. The cytologic outcomes of pleural lavage according to histologic type, pathologic stage, status of lymph node metastasis, pleural involvement of the tumor, lymphatic permeation by the tumor, vascular involvement by the tumor, preoperative value of serum CEA, sex, and age are shown in Table 1. Positive cytologic findings were observed much more frequently in patients with adenocarcinoma (6.9\% [43/622]). Few patients with squamous cell carcinoma had positive cytologic findings (0.6\% [2/339]), and neither patients with large cell carcinoma nor those with adenosquamous carcinoma had positive results. Even in pathologic stage I disease, 13 (2.2\%) of 587 patients had findings positive for malignancy. Malignant cells were detected in the pleural lavage fluid of 6 $(3.5 \%)$ of 173 patients with stage II disease and $26(10.8 \%)$ of 240 patients with stage III disease. Positive findings were seen more frequently in advanced stages. Malignant cells were detected in the pleural lavage fluid of $20(2.2 \%)$ of 658 patients without nodal involvement, $8(4.7 \%)$ of 171 patients with $\mathrm{N} 1$ disease, and 17 (9.9\%) of 171 patients with $\mathrm{N} 2$ disease. Positive findings were more common in cases of higher involvement of lymph nodes. All patients were histopathologically examined for pleural invasion, lymphatic permeation, and vascular involvement. The rates of positive cytologic findings in patients with pleural, lymphatic, and vascular involvement were $16.7 \%$ (30/180), 10.3\% (33/ $321)$, and $7.5 \%$ (32/429), respectively, although in patients without these factors, the rates were $1.8 \%$ (15/820), $1.8 \%$ 
TABLE 1. Cytologic results of pleural lavage fluid according to clinical and pathologic characteristics

\begin{tabular}{|c|c|c|c|c|}
\hline & \multicolumn{2}{|c|}{ Pleural lavage cytology } & \multirow[b]{2}{*}{ Total $(n=1000)$} & \multirow[b]{2}{*}{$P$ value } \\
\hline & Positive ( $n=45$ ) & Negative $(\mathrm{n}=955)$ & & \\
\hline Adenocarcinoma & $43(6.9 \%)$ & 579 & 622 & .0002 \\
\hline Squamous cell carcinoma & $2(0.6 \%)$ & 337 & 339 & \\
\hline Large cell carcinoma & $0(0)$ & 24 & 24 & \\
\hline Adenosquamous carcinoma & $0(0)$ & 15 & 15 & \\
\hline P-stage I & $13(2.2 \%)$ & 574 & 587 & $<.0001$ \\
\hline P-stage II & $6(3.5 \%)$ & 167 & 173 & \\
\hline P-stage III & $26(10.8 \%)$ & 214 & 240 & \\
\hline NO & $20(3.3 \%)$ & 638 & 658 & .0032 \\
\hline N1 & $8(4.7 \%)$ & 163 & 171 & \\
\hline N2 & $17(9.9 \%)$ & 154 & 171 & \\
\hline$P(-)$ & $15(1.8 \%)$ & 805 & 820 & $<.0001$ \\
\hline$P(+)$ & $30(16.7 \%)$ & 150 & 180 & \\
\hline $\operatorname{Ly}(-)$ & $12(1.8 \%)$ & 667 & 679 & $<.0001$ \\
\hline $\operatorname{Ly}(+)$ & $33(10.3 \%)$ & 288 & 321 & \\
\hline$V(-)$ & $13(2.3 \%)$ & 558 & 571 & .0009 \\
\hline$V(+)$ & $32(7.5 \%)$ & 397 & 429 & \\
\hline CEA (-) & $19(3.1 \%)$ & 600 & 619 & .0005 \\
\hline CEA $(+)$ & $26(6.8 \%)$ & 355 & 381 & \\
\hline Male & $25(3.6 \%)$ & 672 & 697 & .0928 \\
\hline Female & $20(6.6 \%)$ & 283 & 303 & \\
\hline Age $\leq 64$ y & $20(4.4 \%)$ & 430 & 450 & .4598 \\
\hline Age $\geq 65 y$ & $25(4.5 \%)$ & 525 & 550 & \\
\hline
\end{tabular}

$P$-stage, Pathologic stage; $N$, lymph node metastasis; $P$, pleural involvement; $L y$, lymphatic permeation; $V$, vascular involvement.

$(12 / 679)$, and $2.3 \%(13 / 571)$, respectively. The frequency of positive cytologic findings in patients with a higher value of preoperative serum CEA was $6.8 \%$ (26/381), whereas in patients with CEA values within normal limits, it was $3.1 \%$ (19/619). There were 619 men and 381 women, with a mean age of 64.2 years (range, 32-85 years). Cancer cells were found in the pleural lavage fluid of $25(3.6 \%)$ of 697 male patients and in that of $20(6.6 \%)$ of 303 female patients. The findings of cytologic examination were not influenced by age. Overall follow-up ranged from 12 to 192 months, with a median (for patients still alive) of 62 months. The 3- and 5 -year survival rates were $46 \%$ and $28 \%$, respectively, in patients with positive findings of pleural lavage and $75 \%$ and $67 \%$ in patients with negative findings (Figure 1). The survival rate was significantly poorer in patients with positive findings than in those with negative findings $(P<$ $.0001)$. Also, the survival rates for 3 and 5 years were $26 \%$ and $16 \%$, respectively, in patients with macroscopic malignant effusion. These data suggested malignant effusion could contribute to a worse prognosis compared with positive lavage cytologic findings $(P=.0510)$. The patients with pathologic stage I disease are generally assumed to have been cured after complete resection of the tumor because lymph nodes were not involved. However, we found that a considerable number of patients having positive cytologic findings had recurrences despite being given a diagnosis of stage I disease. The 5-year survival rates of patients with stage I disease with negative and positive cytologic findings were $81 \%$ and $43 \%$, respectively (Figure 2 ), indicating that positive cytologic findings were associated with a significantly worse prognosis than negative cytologic findings, even in patients with stage I disease $(P=$ .0009). The survival outcomes should be analyzed not only by means of univariate analysis but also by means of multivariable analysis for the prognostic significance of pleural lavage cytology to be strongly emphasized. Multivariable Cox analysis of patients subjected to lavage cytologic examination demonstrated that pleural lavage cytology $(P=$ .0290), as well as sex $(P=.0291)$, age $(P=.0131)$, CEA level $(P=.0017)$ pathologic stage $(P<.0001)$, lymphatic permeation $(P=.0101)$, and vascular involvement $(P=$ .0237), were independent and significant prognostic determinants (Table 2). Of 45 patients with positive findings of pleural lavage fluid, $29(64.4 \%)$ have already had recurrences (Table 3). Distant metastases (65.5\% [19/29]) were observed more frequently than local recurrences, including pleural recurrences $(34.5 \%$ [10/29]). Even in patients with stage I disease, $6(46.2 \%)$ of 13 patients with positive findings had distant metastases, and 2 (15.4\%) had pleural recurrences.

\section{Discussion}

The real question investigated in this study was whether patients who otherwise appear to have resectable tumors would have a worse prognosis if the pleural lavage cytologic findings were positive. When patients with stage I 


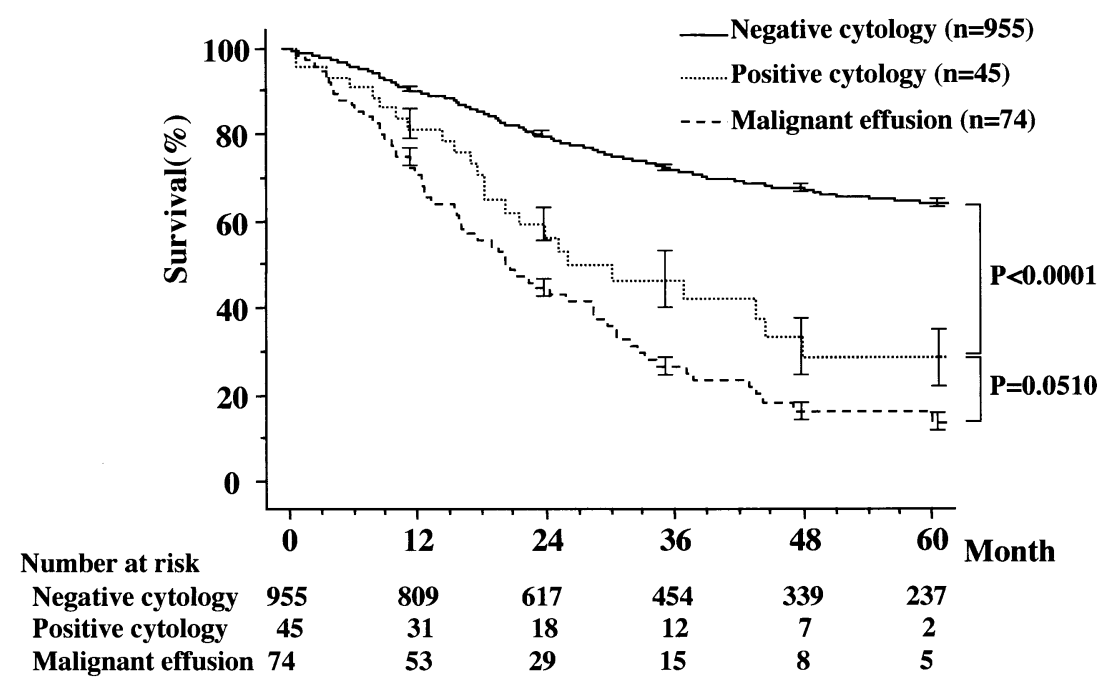

Figure 1. Cumulative survival curves of patients according to cytologic status of pleural lavage fluid. There were 74 patients with malignant effusion undergoing resection of the primary tumor during the same period, but they were excluded from this study. The survival rate of patients with positive cytologic findings was significantly worse than that of patients with negative cytologist findings $(P<.0001)$. In addition, patients with positive cytologic findings had a better (not significant) prognosis than those with malignant effusion $(P=.0510)$.

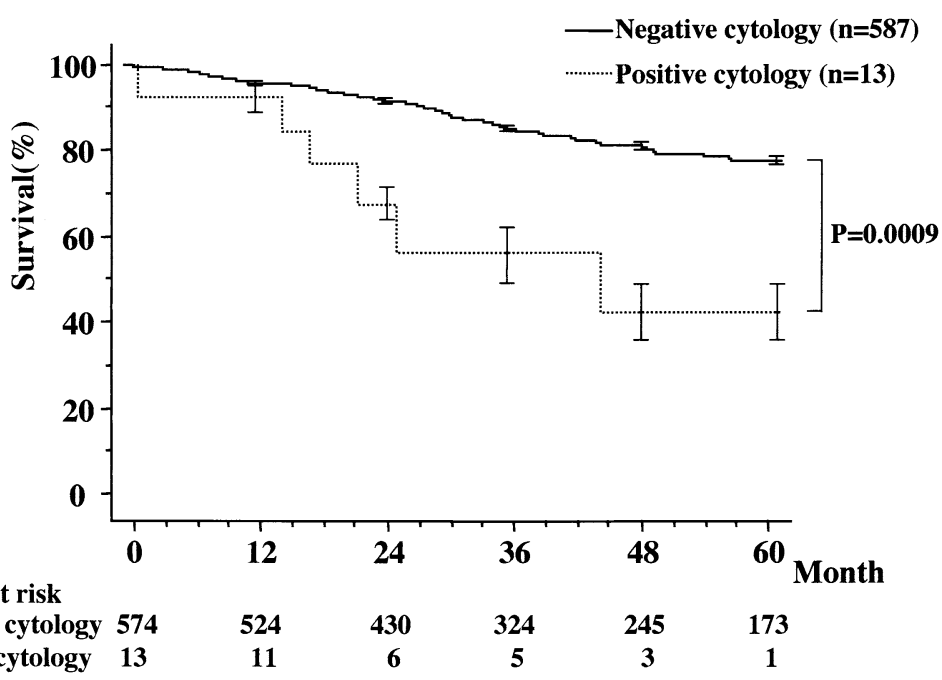

Figure 2. Cumulative survival curves of patients with pathologic stage I disease according to cytologic status of pleural lavage fluid. The survival rate of patients with positive cytologic findings was significantly worse than that of patients with negative cytologic findings $(P=.0009)$.

disease had positive cytologic findings, they showed a significantly poor prognosis, and their 5-year survival was less than $50 \%$ in contrast to more than $80 \%$ for those with negative cytologic findings (Figure 2). In previous reports the prognostic importance of pleural lavage cytology was noted, but its independence in statistics is not known as yet. In this study multivariable analysis demonstrated pleural lavage cytology, as well as sex, age, preoperative serum
CEA value, pathologic stage, lymphatic permeation, and vascular involvement are independent prognostic determinants. Therefore the status of pleural lavage cytology is considered to have a strong effect on survival.

In our previous study the survival rate of patients with positive lavage findings was as poor as that of patients with macroscopic malignant effusion. ${ }^{1}$ However, in the present study patients with positive findings tended to have a better 
TABLE 2. Multivariable analysis of prognostic factors $(n=1000)$

\begin{tabular}{|c|c|c|c|c|c|}
\hline Factors & Unfavorable & Favorable & Risk ratio & $95 \% \mathrm{Cl}$ & $P$ value \\
\hline Sex & Male & Female & 1.550 & $1.171-2.052$ & .0022 \\
\hline Age & $\geq 65$ & $\leq 64$ & 1.330 & $1.062-1.665$ & .0131 \\
\hline CEA & Positive & Negative & 1.431 & $1.143-1.791$ & .0017 \\
\hline Histology & Non-AD & $A D$ & 1.236 & $0.970-1.574$ & .0862 \\
\hline Pathologic stage & II + III & 1 & 3.007 & $2.346-3.854$ & $<.0001$ \\
\hline Lymphatic permeation & Positive & Negative & 1.377 & $1.079-1.759$ & .0101 \\
\hline Vascular involvement & Positive & Negative & 1.307 & $1.171-2.052$ & .0237 \\
\hline Lavage cytology & Positive & Negative & 1.634 & $1.051-2.539$ & .0290 \\
\hline
\end{tabular}

$A D$, Adenocarcinoma.

prognosis than those with malignant effusion. This discrepancy might be due to the difference in the number of patients evaluated and the period of follow-up. In fact, it was of interest to speculate that patients without malignant effusion who showed positive findings of lavage cytology might have a relatively small number of cancer cells in the pleural cavity compared with those with malignant effusion. In other words, it could be concluded that positive lavage cytologic findings are indicative of subclinical pleural dissemination or a stage previous to pleural dissemination.

In this study the link between positive findings on pleural lavage cytology and the histologic type of the tumor should be emphasized. To our surprise, 43 (95.6\%) of 45 patients with positive cytologic findings were given a diagnosis of adenocarcinoma. In addition, 43 (6.9\%) of 622 patients with adenocarcinoma had positive cytologic findings. The presence of subclinical pleural dissemination in a percentage of just about 1 to 15 patients given a diagnosis of adenocarcinoma, even without pleural effusion, is worthy of note. Therefore in patients with lung cancer, especially adenocarcinoma, cytologic examination of pleural lavage fluid should be performed routinely before beginning curative resections and when determining the final stage. One of the most interesting issues refers to how recurrences occur in patients with just positive pleural lavage cytologic findings, particularly in those who were otherwise given a diagnosis of stage I disease. Our data revealed that distant metastasis developed more frequently than local recurrence, including pleural effusion, only among patients with stage I disease and patients with positive lavage findings. Positive cytologic findings of pleural lavage fluid might suggest aggressive biology of the cancer itself because Buhr and colleagues ${ }^{4}$ reported this indicated tumor spread not only in the chest but in the whole body. At present, the pleural lavage procedure is not commonly performed during the staging or resection of tumors in patients with lung cancer. This study describes the significant independent value of the presence or absence of cancer cells in the pleural lavage fluid of
TABLE 3. Recurrence pattern in patients with positive pleural lavage cytologic findings $(n=45)$

\begin{tabular}{lcccl}
\hline & Stage I & Stage II & Stage III & Total \\
\hline No. of patients & 13 & 6 & 26 & 45 \\
No recurrences & 5 & 4 & 7 & 16 \\
With recurrence & 8 & 2 & 19 & 29 \\
$\quad$ Local (pleural cavity) & $2(2)$ & $1(1)$ & $7(5)$ & $10(8)$ \\
$\quad$ Distant & 6 & 1 & 12 & 19 \\
\hline
\end{tabular}

patients undergoing staging or resection for non-small cell lung cancer, particularly adenocarcinoma.

\section{References}

1. Okada M, Tsubota N, Yoshimura M, Miyamoto Y, Maniwa Y. Role of pleural lavage cytology before resection for primary lung carcinoma. Ann Surg. 1999;229:579-84.

2. Okumura M, Ohshima S, Kotake Y, Morino H, Kikui M, Yasumitsu T. Intraoperative pleural lavage cytology in lung cancer patients. Ann Thorac Surg. 1991;51:599-604.

3. Kondo H, Asamura H, Suemasu K, Goya T, Tsuchiya R, Naruke T, et al. Prognostic significance of pleural lavage cytology immediately after thoracotomy in patients with lung cancer. J Thorac Cardiovasc Surg. 1993;106:1092-7.

4. Buhr J, Berghauser KH, Gonner S, Kelm C, Burkhardt EA, Padberg WM. The prognostic significance of tumor cell detection in intraoperative pleural lavage and lung tissue cultures for patients with lung cancer. J Thorac Cardiovasc Surg. 1997;113:683-90.

5. Ichinose Y, Tsuchiya R, Yasumitsu T, Koike T, Yamato Y, Nakagawa $\mathrm{K}$, et al. Prognosis of nonsmall cell lung cancer patients with positive pleural lavage cytology after a thoracotomy: results of the survey conducted by the Japan Clinical Oncology Group. Lung Cancer. 2002;31:37-41.

6. Dresler CM, Fratelli C, Babb J. Prognostic value of positive pleural lavage in patients with lung cancer resection. Ann Thorac Surg. 1999; 67:1435-9.

7. Kjellberg SI, Dresler CM, Goldberg M. Pleural cytologies in lung cancer without pleural effusions. Ann Thorac Surg. 1997;64:941-4.

8. Riquet M, Badoual C, Le Pimpec Barthes F, Lhote FM, Souilamas R, Hubsch JP, et al. Visceral pleura invasion and pleural lavage tumor cytology by lung cancer: a prospective appraisal. Ann Thorac Surg. 2003;75:353-5.

9. Berger RL, Dargan EL, Huang BL. Dissemination of cancer cells by needle biopsy of the lung. J Thorac Cardiovasc Surg. 1972;63: 430-2.

10. Mountain CF. Revisions in the international system for staging lung cancer. Chest. 1997;111:1710-7. 